\title{
Type of article: Review
}

\section{Protein syntheses and autophagy: in contrast or in synchrony?}

\author{
Francesco Saverio Dioguardi, Carol Chen-Scarabelli ${ }^{1}$, Evasio Pasini ${ }^{2}$, Giovanni Corsetti ${ }^{3}$, Tiziano M \\ Scarabelli ${ }^{4}$.
}

Department of Internal Medicine, University of Cagliari, Cagliari, Italy.fsdioguardi@gmail.com

1 Division of Cardiology, Hunter Holmes McGuire Veterans Affairs Medical Center (VAMC), Richmond, VA 23249, USA.

chenscarabelli@hotmail.com

2"Maugeri Scientific Clinical Institutes", IRCCS, Cardiac Rehabilitation Division, Lumezzane (BS), Italy

evpasini@gmail.com

3 Division of Human Anatomy and Physiopathology, Department of Clinical and Experimental Sciences, University of Brescia (BS), Italy.

giovanni.corsetti@unibs.it

4 Center for Heart and Vessel Preclinical Studies, St. John Hospital and Medical Center, Wayne State University, Detroit, Michigan, USA.

tscarabelli@hotmail.com

Author Contributions: Conceptualization: Francesco Saverio Dioguardi. Validation, data curation and discussion, writing, review and editing: Carol Chen-Scarabelli, Evasio Pasini, Giovanni Corsetti, Tiziano M Scarabelli.

"All authors have read and agreed to the published version of the manuscript."

Conflicts of Interest: "The authors declare no conflict of interest".

Compliance with Ethical Standards. not applicable.

Corresponding author:

Prof. Francesco Saverio Dioguardi

Andress: via Venini 1_Milano, 20127-Italy

Tel and Fax: +39-0238314833, e-mail: fsdioguardi@gmail.com

Keywords: nutrition, cancer, protein synthesis, autophagy, mTOR, AMPK, ATP, AMP, amino acids, sarcopenia. 


\section{Abstract}

Background. Sarcopenia, defined as the loss of skeletal muscle mass and function, is a major clinical problem in many chronic illnesses, in cancer and in the elderly. Exercise and adequate nutrition, peculiarly dependents on availability of essential amino acids, considered the primary strategies for prevention and treatment of protein synthetic deficits, affect both the efficient scavenging of aged and overused protein molecules and the renewal, by maintaining muscular protein synthesis. Many questions still remain about the regulation of protein syntheses and degradation. Degradation of inefficient proteins or organelles is performed by the sum of micro and macro-autophagy plus ubiquitin-proteasome system, activities known as proteostasis, necessary to preserve and promote protein masses and consequently, the body's reserves.- However, how protein synthesis is regulated, and how activation of the mTOR complex may modulate and transduce the flow of information provided by exercise and nutrition to balance proteostasis and syntheses, is far from being fully understood. We suggest that-energy production and availability, thus also mitochondria, may have a pivotal role in synchronizing activity and functional outcomes of protein syntheses, and that those syntheses, since higly energy demanding, are main effectors of AMPK dependent autophagy activation by consuming ATP and producing AMP.

Conclusion. While in normal conditions protein syntheses drive autophagy activation by decreasing ATP to AMP ratio, conversely autophagy may be inefficiently activated when chronic both low production and consumption of ATP would result in lowest concentrations of AMP, and therefore both blunted rates of protein syntheses and autophagy would be observed. We suggest that this functional hypothesis may explain sarcopenia in many pathological conditions, as in cancer or in aging muscles.

Key words. mTOR, ATP, protein synthesis, autophagy, amino acids, nutrition, adipose tissue, muscle, sarcopenia, mitochondria. 


\section{Introduction}

The synthesis and renewal of protein structures, known as proteostasis, are intertwined with the two main pathways controlling identification, elimination, and partial recycling of worn-out molecules via: autophagy, the sum of both macro and micro autophagy (ATG) , and the ubiquitin-proteasome system (UPS) [1]. Two tissues, skeletal appendicular muscles and skin comprise, by weight, the first and second major reservoirs of proteins in humans. Due to their multiple dynamic roles in various conditions, muscles should be considered as an organ in a dynamic view of human metabolism requirements of nitrogen, either in health or disease states, so also in cancer [2].

But, when evaluating nitrogen metabolism in the human body, it is often overlooked that the most abundant protein in human body is collagen, with the vast majority stored within the skin dermis, dismantled to refuel energy sources by cancer cells[3].

Notably, the ratio between essential (E) and non-essential (NE) amino acids (AA) both in human or in alimentary proteins is largely $<0.9$. This imbalance underlines that there is a lot yet to be understood in how "messages from environment", that is how materials and energy [4] coming from the world we are living in, are transducers of information first elaborated by DNA and then adaptively modulated through epigenetic responses in those tissues. Indeed, since carbohydrates, lipids and amino acids have similar carbon skeletons, and with the noticeable exception of essential non-saturated fatty acids, sufficient nitrogen amounts can be introduced into mammals metabolism just by amino acids, amino acids provided by food are the most important messengers through which the environment communicates with our body [5]; as well, both oxygen and water should be considered as foods for cells. Therefore, an excess or deficiency of nitrogen in food elicits adaptive mechanisms targeting any tissue or organ and, finally, influencing phenotype and life span [6]. Nutrition also evidently controls the balance between protein synthesis and proteostasis.

An important consideration is that energy expenditure is proportional to physical activity, which increases requirements for maintenance of integrity of the body in basal, resting, healthy conditions. Epidemiological studies have demonstrated that even short, regular periods of non-intense exercise reduce mortality, and 
also prolong the life span of various populations $[7,8]$. On the contrary, inactivity, especially with aging and even for medical reasons, may cause permanent damage to muscles [9], along with muscle mass loss which "is predictive of all-cause mortality in older men regardless of age, BMI, lifestyle, physical performance, health status and body composition"[10].

Those observations suggest that, there are some favorable modifications associated with systematically increasing energy expenditure, but this, in turn, requires to match nutritional intake with exercise-linked requirements, or exercise, peculiarly in the elderly, may not exert the expected advantages [11]

\section{Protein synthesis is required for life, but, how much is enough?}

Protein synthesis is necessary for life, and involves increased activation of the two signaling pathways, extracellular signaling regulated kinase/mitogen activated protein kinase (ERK/MAPK), and mammalian or mechanistic target of rapamycin components (mTORCs commonly identified as C1 and C2), necessary to promote translation, yet a major question is whether protein synthesis is associated with a shorter or longer lifespan. The question arises from studies linked to pharmacological mTORC1 inhibition [12] and observations that caloric restriction reduces mTOR phosphorylation, and the presumed effects on prolonged lifespan. Much has changed from the beginning, and caloric restriction actually has been defined as " a reduction in energy intake below the amount that would be consumed ad libitum (AL) while maintaining adequate intake of essential nutrients" and increased proportions of proteins intakes has been suggested to be necessary for maximal safety [13] . Also, caloric restriction and mTOR inhibition by rapamicin has been shown to be contra-indicated in aging [14].

A main positive effect ascribed to caloric restriction is activation of autophagy due to reduced energy availability and increased AMP concentrations. Reduction of ATP/AMP ratios would in turn both inhibit mTORC1 dependent protein synthesis and activate autophagy [15]. Autophagy is a complex cellular function aimed both to removal of aged structures and to recycle cellular components after dismantling, also funneled into maintaining energy production. Autophagy, in turn, is inhibited when MTORC1 and protein syntheses are fully active and ATP is abundantly available. But, physical exercise, indispensable to 
promote muscular protein synthesis is not only associated to reduction in mortality and extended lifespan, but also to increased autophagy, in evident contradiction with the rapamycin/ caloric restriction and blunted protein synthesis linked predicted benefits. How to solve this conundrum?

The answer is complex since physical exercise, if coupled with an adequate increase in food intake, not only improves protein synthesis throughout the body (muscles, heart, bones, tendons and ligaments), but exercise also needs ATP, and ATP production by oxidation of citrate into mitochondria increases production of reactive oxygen species (ROS) in proportion to the increase in energy requirements (that is of ATP molecules) necessary for matching both demands of workload and energetic costs of protein syntheses. A clue to solving the apparent dichotomy is that ROS are indispensable to activate autophagy, and this activation would link energy production to proteostasis [16], balancing also the availability of substrates at net of energy requirements for protein synthesis. Additionally, nutrition cannot replace exercise in the maintenance of muscle efficiency. This is reinforced by recent research which demonstrated that immobilization activates a block of mTORC1 by blunted phosphorylation of one of its component, DEP domain-containing mTOR interacting protein, DEPTOR, which is only poorly activated by refeeding only, in absence of exercise [17].

\section{Mitochondria, energy availability and autophagy or apoptosis: aging is a complex matter.}

A question still unanswered about senescence driven by mitochondria is what matters more: the number of mitochondria or mitochondrial dysfunction? Mitochondria are the organelle that most efficiently controls energy availability in cells, and certainly energy production and life are strictly connected. Indeed, impairment of energy production is difficult to reverse and results in rapid death[18]. Conversely, it has been shown that by Parkin-mediated mitochondrial autophagy (mitophagy) [19], the generation of viable, but mitochondria-depleted fibroblasts have amelioration of multiple features of senescence [20], as well as those induced by ionizing radiation, such as the production of ROS and the senescence-associated secretory phenotype, the complex of factors including matrix metalloproteinases, growth factors, and proinflammatory cytokines (SASP) [21]. 
Therefore, mTOR, mitochondrial biogenesis, ROS production, autophagy and senescence have been are interrelated, and DNA damage response (DDR) has been described as a part of the "autoregulatory loop" driving senescence triggered through mTOR by mitochondrial biogenesis and maintained by "persistent DDR". Still, those authors recognize that it is principally mitochondrial dysfunction due to impaired or lost mitophagy that would primarily mediate a senescent drive [22].

The accumulation of oxidative damage is the basis of Harman's free radical theory of aging [23].

One of the main sources of ROS in the cell is oxidative phosphorylation within mitochondria, so the free radical theory of aging should be essentially considered a mitochondrial theory of aging, and is so applied from plant seeds [24] to humans, obviously encompassing mouse models [14].

Concurrently, there are studies showing that aging animals have less mitochondrial area/cell volume in muscles and heart [25], and this can be reversed with specific nutritional procedures connected to improved lifespan [26].Excess antioxidants may interfere with longevity [10] and muscle -positive modifications (induced by exercise) as improved insulin sensitivity since also would blunt ROS dependent autophagy [27]. In apparent contradiction of-the studies demonstrating that mitochondrial deletion improved senescence [14], most recently, an inverse correlation was reported between mitochondrial mass and senescence in human T lymphocytes, but indeed, this mass was largely composed of defective mitochondria overproducing ROS [28]. Also, recent studies have demonstrated the necessity of ROSdependent remodeling and mitochondrial biogenesis in order to achieve maximal remodeling and regain of function, following a myocardial infarction [29]. Is it possible to connect those apparently opposite findings? There are two observations that would help in managing this conundrum: first of all, it is evident from all those studies that ROS are indispensable mediators promoting maintenance of any necessary kind of autophagy, thus, including mitophagy. In our opinion, it is not only quantity, i.e. not just number nor volume of mitochondria, but quality of activity and function that matters. Indeed, it is the efficient interconnection between mitochondria efficiency and metabolism that would optimize control of flows among intermediates of different origin (glycolysis or lypolysis) entering citric acid flux, since those ratios may improve or damage energy production both affecting efficiency of cell activities or also ruling 
inflammatory response or DNA damages. This "qualitative" interpretation of mitochondrial function has multiple consequences. For example, an increase in mitochondria number and volume throughout the cell may spread ROS in areas where native anti -oxidative systems are not sufficiently present, thus locally triggering unwanted damages. Additionally, an increased number of mitochondria, possibly a consequence of reduced mitophagy, may be a compensatory response to reduced efficiency. This may occur particularly in conditions of impaired AMPK -dependent activation of sirtuin 1 (SIRT1), a deacetylase in turn controlling activation of the transcriptional regulators PGC-1 $\alpha$ and FOXO1, finally culminating in the transcriptional modulation of mitochondrial and lipid utilization genes, along with oxygen availability coupled with eNOs expression in endothelia, as well as antioxidant availability [19, 20]. Various conditions impairing deacetylation of SIRT1 have been previously described [30], and changes in concentrations of some biochemical mediators may have dramatic consequences on cell behaviors, since metabolic pathways may control activation or inactivation of upstream-controlling gauges. We can simplify the concept by clustering SIRT1 activity when NAD / NADH would be at the opposites. In presence of the lowest concentrations of $\mathrm{NAD}^{+}$, that is when NADH production would be largely prominent, even large numbers of molecules of sirtuins would be ineffective in deacetylating target molecules. Conversely, a small number of sirtuins would be sufficient to elicit a large response if prevalence of $\mathrm{NAD}^{+}$concentrations would be constantly and abundantly maintained. NADH is transformed in $\mathrm{NAD}^{+}$and energy (ATPs) by a well-functioning and active respiratory chain, specifically complex I. Deficiency of $\mathrm{NAD}^{+}$elicits a pseudo-hypoxic state also even in normoxic conditions [31], so NAD deficiencies or excesses following normalization of NAD $/ N A D H$ imbalances are particularly connected with diabetic cardiomyopathy [32], excess alcohol intake [33], and also reduced lifespan [34].

These considerations are summarized as follows: 1 ) some aspects of aging are related to inefficient mitophagy; 2) rather than being mainly considered as a mitochondrial theory of aging, Harmann's free radical theory of aging should have a modified interpretation, considered as an anti-oxidants and autophagy inefficiency-linked theory of aging; and 3) impairment of the balance between activities of both 
mitophagy and biogenesis of renovated and most efficient mitochondria may affect senescence and life span.

Certainly, mTOR activation is connected to mitochondrial biogenesis, and depression of mitochondrial biogenesis is detrimental to cells; thus, mitochondrial activity, ROS production, and naturally occurring antioxidant defenses should be tightly balanced to achieve optimal results [35].

Interestingly, positive activation of mitochondrial biogenesis may occur with metformin. Although metformin can cause an increase in NADH resulting in an alteration in the NAD $/ N A D H$, there is a potent alternative biological instrument for increasing $\mathrm{NAD}^{+} / \mathrm{NADH}$ ratio. Indeed, a reduction of NADH and the same effects obtained by metformin can be achieved by regular and significant implementation of oral EAA intakes which is accompanied by an increased expression and efficiency of cytochrome-C oxidase in cardiomyocytes [36] and in kidneys [37]. There is a recognized need for studies aimed at understanding why mitochondrial biogenesis is possible when there would be loss and failure of efficient mithophagy and focusing on the many modulators that have been found deregulated in cancer cells [38], including in nonneoplastic senescent cells. Noticeably, in normal cells, metformin may stimulate both sirtuin 1 controlled mitochondrial biogenesis as well as EAA. Although it is unknown whether EAA supplementation in normal cells results in activation of the parkin-mediated mitophagy by decreasing inhibitory interaction with and suppressing cytosolic p53 [39], in ob/ob mice-enhancement of autophagy by EAA exposure was described in HCT116 cells [40], and EAA proved to be extremely efficient in restoring mitochondrial efficiency in skeletal muscles and hearts [25] or kidneys [37] of aged animals.

\section{Regulation of protein syntheses: a provocative functional view dictated by lifespan studies.}

-While certain components of aliments positively contribute to protein synthesis [41], autophagy [40], mitochondrial biogenesis, and also reduce renal [37], cardiac and muscular fibrosis and increased life span [26], other components (non essential amino acids: NEAA) acts in an opposite manner, resulting in reduced lifespan [6] 
Indeed, such lifespan studies introduce another level of complexity, since those studies evidenced that it is not just a question of adequate intake of sufficient amounts of EAA, but of intake relative to NEAA introduction, which, if in excess, blunts EAA effects. Also, not all formulations providing EAA exert the same effects. For instance, this important point was emphasized by data from our laboratory, demonstrating that just inverting leucine to isoleucine ratios in some tested EAA composition (without altering any other component) was sufficient to markedly shorten lifespan of mice (unpublished personal data).

Despite eating up to $60 \%$ more food, with quantity to compensate for the lack of quality of AAs, rapid wasting leading to a mortal cachexia occurred in animals fed 30\% EAA and 70\% NEAA, an amount just 15\% less EAA than those provided by the vast majority of alimentary proteins. Animals that had the choice among different pellets, always largely selected the most NEAA-rich, and also the most deadly content [6]. In those animals, a marked alteration of connective tissue and disarrangement of collagen fibers in skin were observed, similar to findings in studies of wound closure $[42,43]$. The rapid mobilization of amino acids from skin in conditions of nitrogen-qualitative malnutrition is important to consider, since there was a very abundant NEAA intake, with the formulation particularly rich in glutamine and arginine, the so called "conditionally essential amino acids". Of notice, very recently it has been shown that availability of sufficiently elevated EAA prevents metabolic deficits of any NEAA, conditionally essential ones included [44].

Collagen, by far the most abundant protein in the body, is vastly composed of NEAAs glycine (33\%) and proline or OH-proline (25\%) [45]. Moreover, within the collagen sequence, the most common AA is lysine, mainly present in its hydroxylated form, $\mathrm{OH}-\mathrm{lysine}$, which is considered the dead end of lysine metabolism [46], no longer useful for protein synthesis, driven to catabolism when released. Therefore, collagen dismantling occurring in malnourished and cancer bearing patients would lead to an overflow of NEAA, that may enter energy production cycles as substrates, but may also blunt favorable epigenetic effects exerted by EAA; thus both EAA and NEAA act as metabokines [47], although often in opposite directions. Based on those observations, two different hypotheses are proposed: 1 ) in the balance of body protein 
disarrangement secondary to insufficient EAA introduction, dermal collagen dismantling may contribute significantly to NEAA increase availability, but only minimally to EAA supply; 2) reduction of EAA/NEAA ratios due to qualitative malnutrition and mobilization of body protein reserves may rapidly modify (epigenetically) metabolic responses in different organs, promoting inhibition of some synthesis and activation of others resulting in growth of cancer [3] and increase damage of some organs [48] already at risk in case of adjuvant chemotherapy.

Our lab has documented a very rapid onset of hypoalbuminemia in healthy animals, despite being fed a diet adequate in calories and a $20 \%$ nitrogen content, but deficient in EAA, providing only $30 \%$ of EAA ( $15 \%$ less than control diets). A rapid wasting with accompanying decline in albumin occurred in the absence of increased proteinuria or other signs of inflammation due to illnesses. Therefore, we think that in the setting of inflammation with subsequent development of malnutrition, treatment of hypoalbuminemia should carefully incorporate metabolic needs and increased intake of EAA, while avoiding NEAA as much as possible.

\section{Autophagy and the balance with protein syntheses}

Since autophagy is a process necessary to any cell, if this process is compromised, cellular function is subsequently affected. Muscle cells have been specifically studied since efficiency of autophagy is indispensable in the promotion of beneficial effects by exercise. As previously stated, autophagy is controlled by energy production and the necessary signaling promoted by ROS is not only functional in autophagy efficiency, but also in the obtaining positive effects of exercise, including enhanced insulin sensitivity [27]. Myocardial training and increased PI3K/AKT/SIRT1/PGC1 $\alpha$ and mitochondrial biogenesisinduced ROS production were shown to be indispensable for optimal remodeling and myocardial protection after ischemic lesions; the balance between autophagy and synthesis of proteins was important to reduce the risks of cardiac fibrosis [49].

$\mathrm{PI3K} / \mathrm{AKT} / \mathrm{SIRT1/PGC1} \alpha$ and mitochondrial biogenesis-induced ROS is well balanced by anti-oxidant production in hearts and muscles of animals treated with EAA formulations; specifically tailored to 
mammals' requirements, diets must also provide significant amounts of leucine, isoleucine and valine, the neutral and branched-chain amino acids [36,37]. On one hand, PI3K/AKT axis activates and coordinates protein synthesis by mTOR [50]; on the other hand, PI3K/AKT activates PGC1 $\alpha$ and mitochondrial biogenesis even in catabolic conditions (denervation, fasting, heart failure, aging and sarcopenia) similar to the effect of constitutively expressed FOX-03 [51]. Those elements are connected by the extreme energy requirements of protein synthesis processes, 4 ATPs any peptidic bond between two AAs [52], and a decline in the ATP/AMP ratio activates AMPK which in turn, blunts synthesis and activates both autophagy and mitophagy functional to maximizing optimal energy availability [53]. Therefore, mTOR activation promotes also its own inactivation continuously alternated to re-activation, and rhythm is given to syntheses by patterns of energy production. When evaluating mTOR and AMPK phosphorylations, we suggest consideration of-median values for calculation as too often inappropriate, since it would underestimate the tightly tuned dynamic alternance of states of activation and inactivation forcing the clouds of results in a switched on-switched off interpretation of data. To further support this view, recent findings on a major component of mTOR, mTORC2, which seems to have an mTORC1-independent capacity of triggering autophagy when activated by deficiencies of some AA, particularly deficiencies in glutamine, a NEAA easily produced into any cell when mitochondrial citric acid cycle would be anaplerotic [54]. Since, alimentary proteins always have a EAA/NEAA ratio significantly lowest than 0,9 [55], it is important to consider the evidence of prolonged cohort survival observed in both mice fed only EAA since the end of lactation [37], and in old animals fed for most of their lives with laboratory pellets and thus fed predominantly EAA (EAA/NEAA > 1) only in the final part of their lives [56].In our viewpoint since dietary modifications have important effects on animal phenotype and lifespan, focus on a particular target is warranted, with our focus on the expression of glutamylprolyl-tRNA synthetase (EPRS), a critical mTORC1 S6K effector, which correlates inversely to either body weight by reducing adipose tissue, and also to increased life span. Of interest, EPRS suppression or reduced phosphorylaton at Ser999 by Eprs knock-in mice bearing phospho-deficient Ser999-to-Ala (S999A) has been shown to correlate with reduced binding, 
translocation to plasma membrane and activation of fatty acid transport protein 1 (FATP1), which regulates uptake of long chain fatty acids, thus linking mTORC1-S6 kinase to phenotype and lifespan [57]. Glutamine is a most abundant AA provided by food proteins, and of notice the effects of a glutaminedeprived, EAA-based diets are similar to those observed on abdominal white adipose tissue of animals with inefficient EPRS phopsphorylation at Ser999. Since EAA have even most pronounced effects, future studies should explore whether there is a relationship between EPRS expression and phosphorylation and reduced uptake of fatty acid and/or reduced syntheses of triglycerides in animals fed only EAA. In agreement with the hypothesis that glutamine controls fatty acid synthesis in adipose tissue, glutamine deprivation increased expression of glutamino synthase [58]. The gene encoding glutamine synthetase (GS or GLUL, glutamate-ammonia ligase) has been identified as a novel transcriptional target of the signaling cascade activated by growth factors, glutamine deprivation surprisingly increased levels of glutamine synthetase, which in turn inhibits mTOR signaling by blunting its lysosomal translocation. Also, it was observed that growth factor signaling through PI3K/AKT may regulate transcriptional programs through inhibitory phosphorylation of Forkhead Box O (FOXO) transcription factors. Conversely, FOXO activation induces autophagosome formation and autophagic flux in a glutamine synthetase-dependent manner [58]. Autophagy is important for maintaining cell survival during conditions of growth factor and nutrient deprivation since inhibition of autophagy induces cell death. However, as we have demonstrated, uncoupling autophagy and ubiquitin-proteasome pathways may trigger rapidly apoptotic death in most rapidly-duplicating cancer cells [40]. Therefore, GS expression and increased ATG are associated with activation of FOXO transcription, particularly with FOXO3 binding to GS-promoter to increase its transcription. Indeed, Van der Vos and Coffer, 2012 [58] have reported an apparently contrasting observations: addition of glutamine to nutrient-starved cells also increases GS expression. This is an interesting finding, worthy of further exploration, since we have studied the amount of GS in the liver of 12 animals, six animals fed by EAA only and six fed laboratory pellet, and found that GS was markedly reduced by EAA feeding $(P<0.02)$, while glutaminase (an enzyme controlling the first step of metabolism of glutamine toward alpha-ketoglutarate) was unaffected (unpublished results kindly provided by Laboratory 
of Dioguardi N , Istituto Clinico Humanitas, Milano, Italy, 2016). These observations not only suggest that both that mTORC1 and FOXOs activities may be sensible and modulated by imbalances among stoichiometric ratios of EAA and NEAA, but also that mTORC2 could be activated by reduced glutamine availability in conditions of elevated plasma EAA/NEAA ratios, when probably either FOXO transcription and autophagy activity are unhooked by the unbalanced ratio between abundant EAA and scarce glutamine availability. Another option is that under conditions of excess NEAA or excess of only one of the amino acids that are most efficient in activating translation and transcription, this excessively elevated concentration needs to be balanced by rapid availability of all other components necessary for protein syntheses. Interestingly, the knockdown of glutaminase and glutamate dehydrogenase, (which together convert glutamine into alpha-ketoglutarate), also inhibited mTOR signaling. Accordingly, increased levels of alpha-ketoglutarate were reported to activate mTOR by increasing GTP loading of RRAGB and lysosomal translocation, resulting in mTORC1 reactivation [58]. On the contrary, in myotubes, excess glutamine was shown to suppress GS expression [59].

Thus, a rationale explaining the impact of -glutamine intake on availability, and in turn the effects on many aspects of metabolism, including how much a feedback mechanism on syntheses is involved, remains to be elucidated. We surmise that inhibition of glutamine synthesis in conditions of high intake and absorption would be mandatory, due to high cerebral toxicity of glutamine [60], and at normal levels of intake, there is no net absorption of glutamine or glutamate, thus, the extensive body glutamine pool is synthesized de novo in physiological settings[61]. Subsequently, an in vivo study in humans would be worthwhile, to examine both whether excess glutamine absorption, overwhelming intestinal efforts to prevent it would reduce GS expression, as we observed in animals fed diets rich in NEAA, and also if glutamine-deficient EAA-based diets would improve in the liver as well as in muscles the BCAA-based glutamine synthesis, while restraining adipose tissue development and prolonging lifespan, as we observed in animal setting $[6,56]$.

\section{Authors view and future fields of research.}


Autophagy and protein synthesis are dynamically related processes, ruled both by concentrations of ATP, and by levels of ATP consumption, so also by energy utilization consequent AMP concentrations raise. Changes in concentrations of ATP and AMP establish depth, frequency and modulation of functions, controlling tightly regulated mTORC1 and AMPK reciprocal intensity of inhibition and activation periods. Thus, the inversion of EAA/NEAA usual ratios provided by foods, and in conditions of EAA/NEAA $>$ or $>>1$, may provide a metabolic strain to which cells would respond significantly. Since activation of protein synthesis is promoted by abundance of EAA, the process may be restrained by the reduced availability of enough NEAA necessary to complete synthesis. Therefore, this sort of "positive nitrogen strain" linked to largest EAA availability would elicit rising consumption of ATP and activation of autophagy to match NEAA need, similarly activated in the setting of the lack of EAA. This would likely further modulated both by ATP to AMP shift related to protein synthesis and linked to the huge energetic costs of the many peptidic bonds necessary for performing huge protein syntheses, as well as by the MTORC1 independent mTORC2 signaling to autophagy described by Vlahakis et al [62] , who demonstrated mTORC2 being a positive regulator of autophagy through its downstream target kinase, Ypk1. Vlahakis et al also demonstrated that AA starvation, specifically of those AA required for auxotrophy, induces autophagy in a manner independent of TORC1 activity and instead requires the general AA control (GAAC) response regulated by the elF $2 \alpha$ kinase, Gcn2 , via the Ca2+-dependent phosphatase calcineurin (which was established as being a negative regulator of the GAAC response and autophagy). Furthermore, knockdown of activating transcription factor 4, the major transcription factor in the GAAC pathway, as well as of SLC7A5, a leucine transporter, caused impaired mTOR reactivation and much higher levels of autophagy [62].

\section{Conclusion}

Protein synthesis and autophagy are tightly regulated processes and alternate reciprocal functions in cells. Protein synthesis, although regulated at various levels, some yet to be elucidated, provides an intrinsic message activating autophagy with consumption of enormous amounts of ATP necessary for completing all peptidic bonds and to accomplish synthesis of -many proteins, especially those of high molecular weight. Large proteins, as enzymes, are formed by thousands of AAs linked at the cost of huge energy expenditure. ATP and ADP consumption, and the resultant increase in AMP concentrations are therefore main effectors of AMPK phosphorylation and activation of autophagy. Thus, we propose that synthesis of proteins controls 
increased production of a second messenger, AMP, in turn triggering autophagy activation and then blunting syntheses following a reduction in energy rich compounds and necessary to replenish ATP availability. Autophagy and the EAA/NEAA ratios are in continuous and tightly coordinated dynamical balance with protein synthesis, since EAA are the main promoters of protein synthesis while NEAA comprise the vast majority of protein sequences in mammals. The long term consequences of those dynamics, that in vitro have been shown to be linked to induction of apoptosis in cancer cells [40], would be evaluated at best by observing cohort lifespan and evaluating the specific changes in organs after prolonged intakes of selected AA formulations $[6,37,56]$. 


\section{References}

${ }^{1}$ Korovila I, Hugo M, Castro JP, Weber D, Höhn A, Grune T, Jung T. Proteostasis, oxidative stress and aging. Redox Biol. 2017 Oct;13:550-567. doi: 10.1016/j.redox.2017.07.008. Epub 2017 Jul 12. PMID: 28763764; PMCID: PMC5536880.

${ }^{2}$ Wolfe RR. The underappreciated role of muscle in health and disease. Am J Clin Nutr. 2006. 84: 475-482.

${ }^{3}$ Phang JM, Donald SP, Pandhare J, Liu Y. The metabolism of proline, a stress substrate, modulates carcinogenic pathways. Amino Acids. 2008. 35: 681-690; PMID:18401543.

${ }^{4}$ Miller JL, Miller JG. Greater than the sum of its parts. II. Matter-energy processing subsystems. Behav Sci. 1993. Jan;38(1):1-73.

${ }^{5}$ Dioguardi FS. Wasting and the substrate-to-energy controlled pathway: a role for insulin resistance and amino acids. Am J Cardiol 2004;93s:6A-12A

${ }^{6}$ Romano C, Corsetti G, Flati V, Pasini E, Picca A, Calvani R, Marzetti E, and Dioguardi FS. Influence of Diets with Varying Essential/Nonessential Amino Acid Ratios on Mouse Lifespan. Nutrients. 2019. 11,6. pii: E1367. doi: $10.3390 /$ nu11061367.

${ }^{7}$ Wen CP, Wai JP, Tsai MK, Yang YC, Cheng TY, Lee MC, Chan HT, Tsao CK, Tsai SP, Wu X. Minimum amount of physical activity for reduced mortality and extended life expectancy: a prospective cohort study. Lancet. 2011. 378, 9798:12441253. doi: 10.1016/S0140-6736(11)60749-6.

${ }^{8}$ Hupin D, Roche F, Gremeaux V, Chatard JC, Oriol M, Gaspoz JM, Barthélémy JC, Edouard P. Even a low-dose of moderate-to-vigorous physical activity reduces mortality by $22 \%$ in adults aged $\geq 60$ years: a systematic review and meta-analysis. Br J Sports Med. 2015. 49: 1262-1267.

${ }^{9}$ Kortebein P, Symons TB, Ferrando A, Paddon-Jones D, Ronsen O, Protas E, Conger S, Juan Lombeida J, Wolfe R and Evans WJ. Functional Impact of 10 Days of Bed Rest in Healthy Older Adults. J Gerontology: MEDICAL SCIENCES. 2008. 63A, 10: 1076-1081

${ }^{10}$ Szulc P, Munoz F, Marchand F, Chapurlat R, and Delmas PD. Rapid loss of appendicular skeletal muscle mass is associated with higher all-cause mortality in older men: the prospective MINOS study. Am J Clin Nutr. 2010. 91: 12271236.

${ }^{11}$ Carlsson M, Littbrand H, Gustafson Y, Lundin-Olsson L, Lindelöf N, Rosendahl E, Håglin L. Effects of high-intensity exercise and protein supplement on muscle mass in ADL dependent older people with and without malnutrition: a randomized controlled trial. J Nutr Health Aging. 2011 Aug;15(7):554-60. doi: 10.1007/s12603-011-0017-5. PMID: 21808934.

12 Papadopoli D, Boulay K, Kazak L et al. mTOR as a central regulator of lifespan and aging [version 1; peer review: 3 approved]. F1000Research 2019, 8(F1000 Faculty Rev):998 (https://doi.org/10.12688/f1000research.17196.1)

${ }^{13}$ Dorling JL, van Vliet S, Huffman KM, Kraus WE, Bhapkar M, Pieper CF, Stewart T, Das SK, Racette SB, Roberts SB, Ravussin E, Redman LM, Martin CK; CALERIE Study Group. Effects of caloric restriction on human physiological, psychological, and behavioral outcomes: highlights from CALERIE phase 2. Nutr Rev. 2021 Jan 1;79(1):98-113. doi: 10.1093/nutrit/nuaa085. PMID: 32940695; PMCID: PMC7727025.

${ }^{14}$ Goldberg EL, Romero-Aleshire MJ, Renkema KR, Ventevogel MS, Chew WM, Uhrlaub JL, Smithey MJ, Limesand KH, Sempowski GD, Brooks HL, Nikolich-Žugich J. Lifespan-extending caloric restriction or mTOR inhibition impair adaptive immunity of old mice by distinct mechanisms. Aging Cell. 2015 Feb;14(1):130-8. doi: 10.1111/acel.12280. Epub 2014 Nov 26. PMID: 25424641; PMCID: PMC4326902.

15 Tee AR. The target of rapamycin and mechanisms of cell growth. Int J Mol Sci. 2018 Mar 16;19(3):880. doi: 10.3390/ijms19030880.

${ }^{16}$ Ristow M, Schmeisser S. Extending life span by increasing oxidative stress. Free Radical Biology \& Medicine 51 (2011) 327-336.

${ }^{17}$ Shimkus KL, Jefferson LS, Gordon BS, Kimball SR. Repressors of mTORC1 act to blunt the anabolic response to feeding in the soleus muscle of a cast-immobilized mouse hindlimb. Physiol Rep. 2018 Oct;6(20):e13891. doi: 10.14814/phy2.13891.

${ }^{18}$ Chacko B, Peter JV. Antidotes in Poisoning. Indian J Crit Care Med 2019;23(Suppl 4):S241-S249.

${ }^{19}$ Narendra D, Tanaka A, Suen DF, Youle RJ. Parkin is recruited selectively to impaired mitochondria and promotes their autophagy.2008. J Cell Biol 183: $795-803$

${ }^{20}$ Correia-Melo C, Marques FD, Anderson R, Hewitt G, Hewitt R, Cole J, Carroll BM, Miwa S, Birch J, Merz A, Rushton MD, Charles M, Jurk D, Tait SW, Czapiewski R, Greaves L, Nelson G, Bohlooly-Y M, Rodriguez-Cuenca S, Vidal-Puig A, Mann D, Saretzki G, Quarato G, Green DR, Adams PD, von Zglinicki T, Korolchuk VI, Passos JF. Mitochondria are required for pro-ageing features of the senescent phenotype. EMBO J. 2016 Apr 1;35(7):724-42. doi:

10.15252/embj.201592862. Epub 2016 Feb 4.

${ }^{21}$ Salama R, Sadaie M, Hoare M, Narita M. Cellular senescence and its effector programs.

Genes Dev 2014. 28: $99-114$ 
${ }^{22}$ Herranz H, Gil J. Mitochondria and senescence: new actors for an old play. EMBO J. 2016. 35,7: 701-702.

${ }^{23}$ Harman, D. Free radical theory of aging: an update: increasing the functional lifespan. Ann. N. Y. Acad. Sci. 2006, 1067, 10-21.

${ }^{24}$ Ratajczak E, Arleta Małecka A, Ciereszko I ,M. Staszak AM. Mitochondria Are Important Determinants of the Aging of Seeds. Int. J. Mol. Sci. 2019, 20, 1568; doi:10.3390/ijms20071568

${ }^{25}$ Corsetti G1, Pasini E, D'Antona G, Nisoli E, Flati V, Assanelli D, Dioguardi FS, Bianchi R. Morphometric changes induced by amino acid supplementation in skeletal and cardiac muscles of old mice. Am J Cardiol. 2008 Jun 2;101(11A):26E-34E. doi: 10.1016/j.amjcard.2008.02.078.

${ }^{26}$ D’Antona, G.; Ragni, M.; Cardile, A.; Tedesco, L.; Dossena, M.; Bruttini, F.; Caliaro, F.; Corsetti, G.; Bottinelli, R.; Carruba, M.O.; et al. Branched-chain amino acid supplementation promotes survival and supports cardiac and skeletal muscle mitochondrial biogenesis in middle-aged mice. Cell Metab. (2010) 12:362-72. doi: 10.1016/j.cmet.2010.08.016 ${ }^{27}$ Ristow M, Zarsea K, Oberbach A, Kloting N, Birringer M, Kiehntopf M, Stumvoll M, C. KahneR, and Matthias Bluher M. Antioxidants prevent health-promoting effects of physical exercise in humans. PNAS. 2009., 106, 21:8665-8670.

${ }^{28}$ Callender LA, Carroll EC, Bober EA, Akbar AN, Solito E, Henson SM. Mitochondrial mass governs the extent of human T cell senescence. Aging Cell. 2020 Feb;19(2):e13067. doi: 10.1111/acel.13067

${ }^{29}$ Jia D, Hou L, Lv Y, Xi L, Tian Z. Postinfarction exercise training alleviates cardiac dysfunction and adverse remodeling via mitochondrial biogenesis and SIRT1/PGC-1 $\alpha /$ PI3K/Akt signaling. J Cell Physiol. 2019;234:23705-23718. ${ }^{30}$ Webster BR, Scott I, Traba J, Han K, Sack MN. Regulation of autophagy and mitophagy by nutrient availability and acetylation. Biochimt Biophys Acta. 1841 (2014) 525-534

${ }^{31}$ Lee CF, Caudal A, Abell L, Nagana Gowda GA, Tian R. Targeting NAD ${ }^{+}$Metabolism as Interventions for Mitochondrial Disease. Sci Rep. 2019 Feb 28;9(1):3073. doi: 10.1038/s41598-019-39419-4.

${ }^{32}$ Berthiaume JM, Kurdys JG, Muntean DM, Rosca MG.Mitochondrial NAD ${ }^{+}$NADH Redox State and Diabetic Cardiomyopathy. Antioxid Redox Signal. 2019 Jan 20;30(3):375-398. doi: 10.1089/ars.2017.7415.

${ }^{33}$ Tedesco L, Corsetti G, Ruocco C, Ragni M, Rossi F, Carruba MO, Valerio A, NisoliE. A specific amino acid formula prevents alcoholic liver disease in rodents. Am J Physiol Gastrointest Liver Physiol 314: G566-G582, 2018.

${ }^{34}$ Castro-Portuguez R, Sutphin GL. Kynurenine pathway, NAD+ synthesis, and mitochondrial function: Targeting tryptophan metabolism to promote longevity and healthspan. Exp Gerontol. 2020 Apr;132:110841. doi: 10.1016/j.exger.2020.110841. Epub 2020 Jan 16.

${ }^{35}$ Dalle Pezze P, Nelson G, Otten EG, Korolchuk VI, Kirkwood TBL, et al. (2014) Dynamic Modelling of Pathways to Cellular Senescence Reveals Strategies for Targeted Interventions. PLoS Comput Biol 10(8): e1003728.

doi:10.1371/journal.pcbi.1003728

${ }^{36}$ Tedesco L, Rossi F, Ragni M, Ruocco C, Brunetti C, Carruba MO, Torrente Y, Alessandra Valerio A, and Nisoli E. A Special Amino-Acid Formula Tailored to Boosting Cell Respiration Prevents Mitochondrial Dysfunction and Oxidative Stress Caused by Doxorubicin in Mouse Cardiomyocytes. Nutrients 2020, 12, 282; doi:10.3390/nu12020282.

${ }^{37}$ Corsetti G, Stacchiotti A, D' Antona G, Nisoli E, Dioguardi FS, Rezzani R. Supplementation with essential amino acids in middle age maintains the health of rat kidney. Int J Immunopathol Pharmacol. 2010. 23(2):523-533.

${ }^{38}$ Drake LE, Springer MZ, Poole LP, Kim CJ, Macleod KF. Expanding perspectives on the significance of mithophagy in cancer. Semin Cancer Biol. 2017 Dec;47:110-124. doi: 10.1016/j.semcancer.2017.04.008.

${ }^{39}$ Song YM, Lee WK, Lee Y-h, Kang ES, Cha B-S and Lee B-W. Metformin Restores Parkin-Mediated Mitophagy, Suppressed by Cytosolic p53. Int. J. Mol. Sci. 2016, 17, 122; doi:10.3390/ijms17010122.

${ }^{40}$ Bonfili L, Cecarini V, Cuccioloni M, Angeletti M, Flati V, Corsetti G, Pasini E, Dioguardi FS, Eleuteri AM. Essential amino acid mixtures drive cancer cells to apoptosis through proteasome inhibition and autophagy activation. FEBS J. 2017 Jun;284(11):1726-1737. doi: 10.1111/febs.14081. Epub 2017 May 11.

${ }^{41}$ Volpi E, Kobayashi H, Sheffield-Moore M, Mittendorfer B, and Wolfe RR. Essential amino acids are primarily responsible for the amino acid stimulation of muscle protein anabolism in healthy elderly adults. Am J Clin Nutr 2003;78:250-258.

${ }^{42}$ Corsetti G, Romano C, Pasini E, Marzetti E, Calvani R, Picca A, Flati V, Dioguardi FS. Diet enrichment with a specific essential free amino acid mixture improves healing of undressed wounds in aged rats. Exp Gerontol. 2017 Oct 1;96:138-145. doi: 10.1016/j.exger.2017.06.020. Epub 2017 Jun 29

${ }^{43}$ Corsetti G , Pasini E, Flati V, Romano C, Rufo A and Dioguardi FS. Aging Skin: Nourishing from the Inside Out, Effects of Good Versus Poor Nitrogen Intake on Skin Health and Healing. in: Farage MA, Miller KW and Maibach HI. Textbook of Aging Skin. Pages 1619-1629. Springer Berlin Heidelberg , Berlin, Heidelberg. DOI: 10.1007/978-3-64227814-3 135-1

${ }^{44}$ Cooper L, Ball RO, Pencharz PB, Sakai R, Elango R. Dispensable Amino Acids, except Glutamine and Proline, Are Ideal Nitrogen Sources for Protein Synthesis in the Presence of Adequate Indispensable Amino Acids in Adult Men. J Nutr. 2020 Sep 1;150(9):2398-2404. doi: 10.1093/jn/nxaa180. PMID: 32879983. 
${ }^{45}$ Phang JM, Liu W and Hancock C. Bridging epigenetics and metabolism. Role of non essential amino acids. Epigenetics 8:3, 231-236; March 2013.

${ }^{46}$ Dioguardi FS. Nutrition and skin. Collagen integrity: a dominant role for amino acids.

Clin Dermatol. 2008 Nov-Dec;26(6):636-40. doi: 10.1016/j.clindermatol.2007.09.004.

${ }^{47}$ Naviaux RK. Metabolic features and regulation of the healing cycle-A new model for chronic disease pathogenesis and treatment. Mitochondrion.2019. 46:278-297.

${ }^{48}$ Yang Z, Huang J, Ladeiras D, Yu Y and Ming X-F. Detrimental effects of chronic L-arginine rich food on aging kidney. In press: Front. Pharmacol. | doi: 10.3389/fphar.2020.582155

49 Jia D, Hou L, LV Z, Xi L, Tian Z. Postinfarction exercise training alleviates cardiac dysfunction and adverse remodeling via mitochondrial biogenesis and SIRT1/PGC-1 $\alpha$ /PI3K/Akt signaling. J Cell Physiol. 2019. 234:23705-23718.

${ }^{50}$ Morita M, Gravel S-P, Hulea L, Larsson O, Pollak M, St-Pierre J, Topisirovic I. mTOR coordinates protein synthesis, mitochondrial activity and proliferation. Cell Cycle. 2015. 14,4: 473-480.

${ }^{51}$ Schiaffino S, Dayar KA, Ciciliot C, Blaauw and Sandri M. Mechanism regulating skeletal muscle growth and atrophy. FEBS J. 2013. 280: 4294-4314.

${ }^{52}$ Kaleta S, Schauble S, Rinas U, Schuster S. Metabolic costs of amino acid and protein production in Escherichia coli . Biotechnol. J. 2013, 8, 1105-1114

${ }^{53}$ Hardie DG, Ross FA and Hawley SA. AMPK: a nutrient and energy sensor that maintains energy homeostasis. Nature Rev. Mol Cell Biol. 2012. 13: 251-262.

${ }^{54}$ Liao GY, Lee MT, Fan JJ, Hsiao PW, Lee CS, Su SY, Hwang JJ, Ke FC. Blockage of glutamine-dependent anaplerosis affects mTORC1/2activity and ultimately leads to cellular senescence-like response. Biol Open. 2019 May 16;8(5). pii: bio038257. doi: 10.1242/bio.038257.

${ }^{55}$ Gorissen SH and Witard OC. Characterising the muscle anabolic potential of dairy, meat and plant-based protein sources in older adults. Proceedings of the Nutrition Society (2018), 77, 20-31 doi:10.1017/S002966511700194X.

${ }^{56}$ Corsetti G, Pasini E, Romano C, Calvani R, Picca A, Marzetti E, Flati V and Dioguardi FS. Body Weight Loss and Tissue Wasting in Late Middle-Aged Mice on Slightly Imbalanced Essential/Non-essential Amino Acids Diet. Front. Med. 5:136. doi: 10.3389/fmed.2018.00136

${ }^{57}$ Arif A, Terenzi F, Potdar AA, Jia J, Sacks J, China A, Halawani D, Vasu K, Li X, Brown B, Chen J, Kozma SC, Thomas G, Fox PL. EPRS is a critical mTORC1-S6K1 effector that influences adiposity in mice. Nature. 2017. 542(7641):357361.doi:10.1038/nature21380

${ }^{58}$ van der Vos KE, Eliasson P, Proikas-Cezanne T, Vervoort SJ, van Boxtel R, Putker M, et al. Modulation of glutamine metabolism by the PI(3)K-PKB-FOXO network regulates autophagy.

Nat Cell Biol 2012; 14:829-37; PMID:22820375; http://dx.doi.org/10.1038/ncb2536

${ }^{59}$ Huang Y-F, Wang Y, and Malcolm Watford M. Glutamine Directly Downregulates Glutamine Synthetase Protein Levels in Mouse C2C12 Skeletal Muscle Myotubes. J. Nutr. 137: 1357-1362, 2007.

${ }^{60}$ Dhaher R, Chen EC, Perez E, Rapuano A, Sandhu MRS, Gruenbaum SE, Deshpande K, Dai F, Zaveri HP, Eid T. Oral glutamine supplementation increases seizure severity in a rodent model of mesial temporal lobe epilepsy. Nutr Neurosci. 2020 Jan 3:1-6. doi: 10.1080/1028415X.2019.1708568.

${ }^{61}$ Watford M. Glutamine Metabolism and Function in Relation to Proline Synthesis and the Safety of Glutamine and Proline Supplementation. J. Nutr. 138: 2003S-2007S, 2008.

${ }^{62}$ Vlahakis A, Graef M, Nunnari J and Powers T. TOR complex 2-Ypk1 signaling is an essential positive regulator of the general amino acid control response and autophagy. PNAS. 2014. 111,29: 10586-10591 